\title{
Galaxy Evolution in the Optical and Submillimeter
}

\author{
Simon Lilly \\ Department of Astronomy, University of Toronto, Toronto, Ontario, \\ M5S 3H8, Canada
}

\begin{abstract}
I review the emerging picture of galaxy formation and evolution that is coming from observations in the optical waveband and in the submillimeter waveband. In the former I look at the work of several groups but in the latter I focus on the results from the Canada-UK Deep Submillimeter Survey (CUDSS), an 850 micron survey of about 100 square arcmin of sky carried out with SCUBA to a limiting flux of $3 \mathrm{mJy}$. I discuss what we do and do not know about the sources responsible for emitting a significant fraction of the extragalactic background at 850 microns.
\end{abstract}

\section{Discussion}

Jim Felten: I'm looking at the first point of your summary and trying to boil it down. Can you resolve (or sharpen up) the paradox raised the other day by Jim Peebles: If the large galaxies are already in place by $z=1$, and if star-formation histories show that quite a lot of formation has occurred in the interval $0<z<1$, then where have these stars formed, and where are they now? Not in the large galaxies? 